\title{
TRISMUS IN AMBULATORY SETTING: A CLINICAL CASE
}

C. Monteiro ${ }^{1}$, A. Gouveia ${ }^{1}$, S. Gestosa ${ }^{1}$, A. Lares ${ }^{1}$

${ }^{1}$ Centro Hospitalar Universitário do Algarve - Unidade de Faro - Faro (Portugal)

\section{BACKGROUND:}

This is a case report of unsuspected difficult airway managment secondary to difficulty in mouth opening of an anesthetized adult. Trismus was first defined as reduced opening of the jaws specifically caused by mastication muscles spasm. Currently, this term refers to limited mouth opening from any etiology, being encountered with increasing frequency in clinical practice.[1] Most of the earlier reports describe temporomandibular dislocation (TMD) in patients who have had past history of joint dysfunction. However, sometimes it may occur even in normal temporomandibular junction.[2]

\section{CASE REPORT:}

A male patient, 62 years-old, was proposed to hydrocele repair in ambulatory setting. Previous medical history included smoking and saphenectomy. Preoperative airway evaluation didn't anticipate a difficult airway (mallampati class 2, no restriction in mouth opening or cervical mobility) and was graded ASA II. After pre-oxygenation, intravenous (IV) fentanyl and propofol were administered. An attempt to open the patient's mouth was made after 1 min but the teeth were closely approximated, and the mouth could not be opened. A second attempt 1 min later by the senior anesthesiologist and after proper anesthesia depth verification and simple airway maneuvers also failed to open the mouth. After failure to insert a laringeal mask, the patient was ventilated through mask ventilation. Considering that fiberscope and video laringoscope weren't readily available, the surgery was called-off and the patient was awakened.
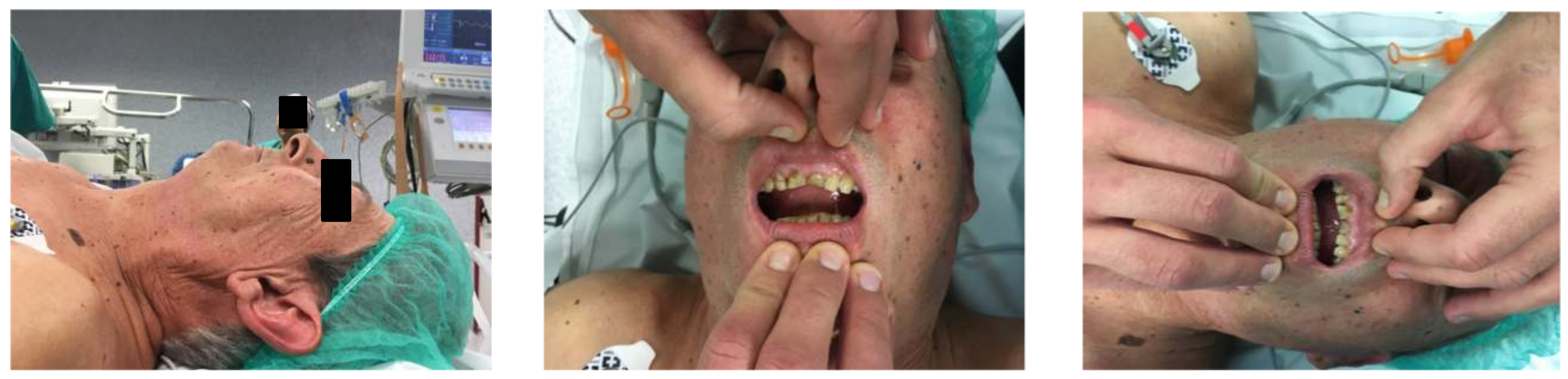

Figures 1, 2 and 3: Lateral and anterior view of patient reduced mouth opening.

\section{DISCUSSION:}

This case report illustrates a potential hazard in airway management during general anesthesia (GA). In severe trismus, it is difficult or even impossible to insert a laryngoscope, an endotracheal tube or an laryngeal mask between the teeth.[2] The anterior translation of the TMJ and jaw-thrust maneuver play an important in mouth opening. The recommended jaw-thrust maneuver to overcome the locked-jaw and obstruction were ineffective in this case.

\section{LEARNING POINTS:}

Ensuring airway protection during GA is the cornerstone of anesthesia practice. This case illustrates that trismus could happen without warning or and can lead to potential life-threatening situations. 